\title{
Wideband Differentially Fed Dual-Polarized Antenna by Using Three-Strip Transmission Lines
}

\author{
Le-Hu Wen, Steven Gao, Qi Luo, Benito Sanz-Izquierdo, Xiaofei Ren, and Jian Wu
}

\begin{abstract}
A new method of designing a wideband differentially fed dual-polarized antenna by using three-strip transmission lines (TS-TLs) is presented. A wideband power divider composed of four TS-TLs is firstly proposed and illustrated by using its equivalent circuit. Based on the proposed power divider, a wideband differentially fed dual-polarized antenna with four in-band resonances is developed. To enhance the outof-band selectivity, two radiation nulls with quasi-elliptic response are obtained by utilizing the specifically designed structures in the TS-TL based power divider. The first radiation null at the lower out-of-band is produced by the resonance from the center strip of TS-TL and shorting coaxial cable. The second radiation null at the upper out-of-band is produced by the resonance from the slot formed by the dipole arm and the outer strip of TS-TL. Combining with the high $2^{\text {nd }}$-order harmonic suppression owing to the differentially fed method, the presented differentially fed antenna is demonstrated that it not only has high inband selectivity covering 1.67-2.91 GHz, but also has high out-of-band suppressions of $33.8 \mathrm{~dB}$ and $20.6 \mathrm{~dB}$ over the frequency bands of 0.6-1 GHz and 3.3-5 GHz. In addition, stable gain and beamwidth are achieved for base station applications.
\end{abstract}

Index Terms-Differentially fed antenna, dual-polarized antenna, power divider, three-strip transmission line.

\section{INTRODUCTION}

As the development of modern wireless communication systems, such as base stations, radars, satellites, etc., dual-polarized antennas have been drawn wide attentions because of the advantages of reduced multi-path effect and increased channel capacity benefiting from the polarization diversity technique [1]. An easy way to realize dual-polarization is exciting two orthogonal resonating modes on a square patch by using either the direct probe feed [2], capacitive probe coupling [3], aperture coupling [4]-[5], or the mixed feed methods [6]-[7]. To obtain wide impedance bandwidth and stable radiation characteristic, crossed dipoles combined with a planar reflector are utilized to develop dual-polarized antennas [8]-[10]. With the combination of electric dipole and magnetic dipole, dualpolarized multi-resonated antennas are developed [11]-[14]. In addition, by introducing the integrated crossed baluns as the feed lines, it is convenient to develop wideband [15] and dual-band [16] dual-polarized base station antennas. However, little of these antennas are in consideration of the undesired out-of-band interference signals.

Because of the advantages of low noise level, high harmonic rejection, and high mode current suppression, differential electronic components and devices are widely applied in wireless communication systems [17]. Therefore, to directly match these differential components and devices, differentially fed antennas are widely researched [18]-[22]. In this work, a wideband power divider

This work was supported in part by China Research Institute of Radiowave Propagation, in part by EPSRC grants EP/N032497/1, EP/P015840/1, and EP/S005625/1, and in part by China Scholarship Council.

L.-H. Wen, S. Gao, and B. Sanz-Izquierdo are with the School of Engineering and Digital Arts, University of Kent, Canterbury, CT2 7NT, U.K. (e-mail:1w347@kent.ac.uk)

Q. Luo is with the School of Physics, Engineering and Computer Science, University of Hertfordshire, Hatfield, AL10 9AB, UK.

$\mathrm{X}$. Ren and J. Wu are with the Innovation and Research Center, China Research Institute of Radiowave Propagation, Qingdao, 266107, China. composed of three-strip transmission lines (TS-TLs) is first proposed and illustrated. Detailed analysis shows that the TS-TL based power divider can have wide impedance bandwidth with two reflection zeros under the differential excitation. Compared to the parallel-line based power divider presented in our previous work in [22], the TSTL based power divider has the unique structures and impedance characteristics with the different equivalent circuits.

The unique characteristics of the TS-TL based power divider are further utilized to realize high in-band selectivity with four reflection zeros and high out-of-band suppression with two controllable radiation nulls at both the lower and upper out-of-bands with quasielliptic response. Analyses show that the first lower out-of-band radiation null is produced by the resonance from the center strip of the TS-TL and the shorting coaxial cable, which is realized for high suppression over $0.6-1 \mathrm{GHz}$. The second upper out-of-band radiation null is produced by the resonance from the slot formed by the outer strip of the TS-TL and the dipole arm, which is realized for high suppression over 3.3-3.6 GHz. Combining with the high $2^{\text {nd }}$-order harmonic suppression owing to the differentially fed method, the presented antenna has the high and wide upper out-of-band suppression over 3.3-5 GHz.

The presented antenna keeps simple configuration for practical industrial applications without any traditionally introduced parasitic patches, strips, etched slots, or baluns for the performance enhancement. The measured results demonstrate that the presented differentially fed antenna has a wide impedance bandwidth of 1.67$2.91 \mathrm{GHz}$ for $\left|S_{d d 11}\right|<-15 \mathrm{~dB}$. Moreover, high lower and upper outof-band suppressions of $33.8 \mathrm{~dB}$ over $0.6-1 \mathrm{GHz}$ and $20.6 \mathrm{~dB}$ over 3.3-5 GHz are achieved.

\section{TS-TL BASED POWER DIVIDER}

A power divider composed of four TS-TLs is first proposed as the integrated feed network for the presented antenna. Fig. 1 (a) shows the configuration of the TS-TL based power divider. The power divider is composed of four TS-TLs with two input ports (port 1 and port 2) at the center and four output ports (port 3 to port 6) at the four sides of the structure. Each center ends of the outer strips of the TSTLs are connected together, while the other ends of the outer strips are left open and designed as four output ports. The inner strips of the four TS-TLs at the center are designed as two input ports, while the other ends of the inner strips are left open-circuited. Detailed parameters of the configuration are listed in the caption of Fig. 1 (a).

To illustrate the working principle of the TS-TL based power divider, the proposed power divider under the differential excitation at the input port is analyzed. Fig. 1 (b) shows the simulated Sparameters of the proposed TS-TL based power divider. In the design, the differential source impedances of the input ports are designed as $100 \mathrm{ohm}$. The load impedances of the output ports are designed as the odd-mode characteristic impedance of the transmission line, which is $200 \mathrm{ohm}(\mathrm{D} 1=0.6 \mathrm{~mm}$ and $\mathrm{W} 2=2 \mathrm{~mm}$ ) in the simulation. It can be seen that a deep reflection zero is located at $2.2 \mathrm{GHz}$ with the simulated $\left|\mathrm{S}_{11}\right|<-10 \mathrm{~dB}$ from $1.69 \mathrm{GHz}$ to 2.77 GHz. Equal magnitude transmission is produced from port 1 to port 3 and port 5 respectively across the whole impedance bandwidth. 
Whereas the port 2, port 4, and port 6 are highly isolated from port 1 without power transmission. Owing to the symmetry of the structure, when port 2 is differentially excited, port 4 and port 6 will have the equal outputs, while port 1 , port 3 , and port 5 are highly isolated. These similar results are not included in the figure for brevity.

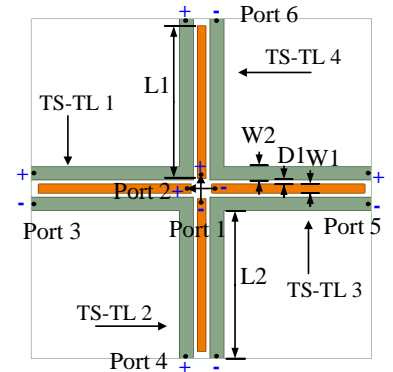

(a)

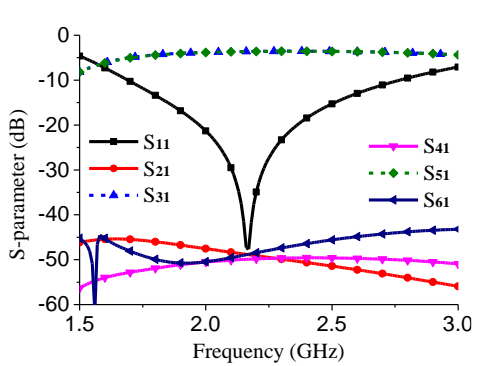

(b)
Fig. 1. (a) Configuration of the proposed power divider composed of four TSTLs. (Parameters in the configuration. W1=1.3 mm, L1=21.5 mm, L2=26 $\mathrm{mm}$.) (b) Simulated S-parameters of the proposed TS-TL based power divider.

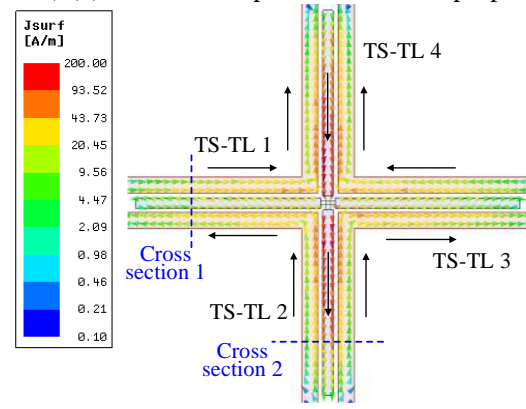

(a)

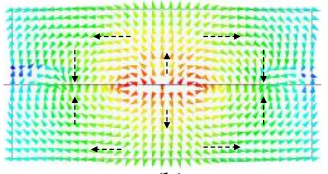

(b)

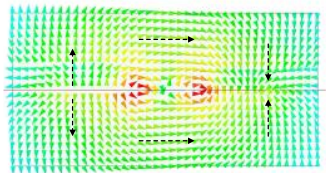

(c)
Fig. 2. Field distributions of the proposed TS-TL based power divider at 2.2 GHz. (a) Surface current distribution. (b) Odd-mode E-field distribution in the cross section 1. (c) Even-mode E-field distribution in the cross section 2.

The field distributions including the surface current distribution and E-filed distributions in the cross sections of the TS-TL are shown in Fig. 2 (a) for the transmission characteristic analysis. As shown in figure, when port 1 is differentially excited, strong current magnitude is observed on the inner strips of TS-TL 1 and TS-TL 3, and two outer strips are coupled with inverse direction current. The current flows on the two outer strips can be used to drive the dipole arms. Differently, two outer strips of TS-TL 2 and TS-TL 4 are coupled with the currents in the same direction, but the direction of them is inverse to current distributed on the inner strips. The current distribution on TS-TL 1 and TS-TL 3 shows that the transmission mode on these two transmission lines is odd mode, and the current distribution on TS-TL 2 and TS-TL 4 shows that the transmission mode on these two transmission lines is the even mode. This can be further demonstrated by the E-field distribution in the cross section 1 and cross section 2 on these two transmission lines, as shown in Fig. 2 (b) and (c). For the E-field distribution in cross section 1 around the TS-TL 1, the E-field comes from the left outer strip, and ends to the right outer strips, which is the odd symmetry. The E-field in cross section 2 comes from the center strip, and ends to the two outer strips respectively, which is the even symmetry.

Owing to the structure symmetry and the even-odd mode transmission characteristic, its equivalent circuit under the differential excitation at port 1 can be obtained and shown in Fig. 3 (a). It is composed of an open-circuited stub with characteristic impedance $2 Z_{e}$ and electric length $\theta_{e}$ and a transmission line with characteristic impedance $Z_{o} / 2$ and electric length $\theta_{o}$. According to the equivalent circuit shown in this figure, the impedance bandwidth of the TS-TL power divider can be further enhanced.

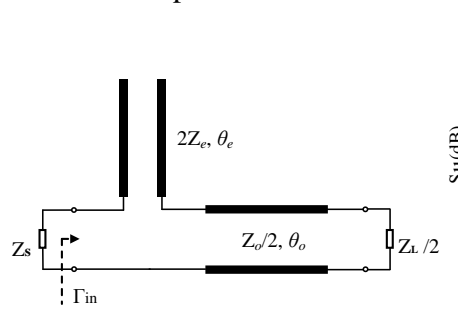

(a)

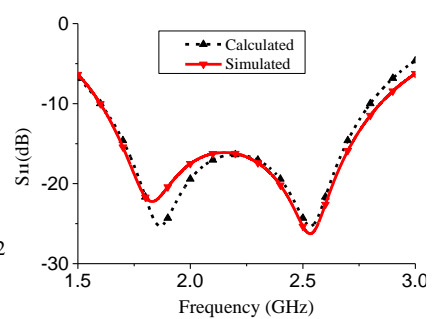

(b)
Fig. 3 (a). Equivalent circuit of the TS-TL based power divider under the differential excitation at port 1 . (b). Calculated and simulated reflection coefficients when $Z_{L}=50 \mathrm{ohm}$ and $Z_{o}=116.4 \mathrm{ohm}$.

In Fig. 3 (a), the series transmission line $\left(Z_{o} / 2, \theta_{o}\right)$ can work as an impedance inverter to increase the impedance bandwidth of the proposed power divider. For example, when $\theta_{e}=\theta_{o}=90^{\circ}$, the input impedance at the input port can be directly calculated as

$$
Z_{\text {in }}=\frac{Z_{o}^{2}}{2 Z_{s} Z_{L}}
$$

Therefore, the input impedance can be adjusted by changing the oddmode characteristic impedance of the TS-TL when the load impedance cannot be directly matched to the odd-mode characteristic impedance of the TS-TL.

As an important feed network for the dual-polarized antenna, the load of the TS-TL based power divider is normally the dipole, and its input impedance is often designed as $50 \mathrm{ohm}$. To match the input impedance of the dipole and get much lower reflection coefficient at the input port, the odd-mode characteristic impedance of the TS-TL should be carefully designed. Fig. 3 (b) shows the simulated reflection coefficient when $Z_{L}=50 \mathrm{ohm}$ and $Z_{o}=116.4 \mathrm{ohm}$ (D1=0.2 $\mathrm{mm}$ and $\mathrm{W} 2=4 \mathrm{~mm}$ ), which is also compared to the calculated result by using the equivalent circuit shown in Fig. 3 (a). As can be seen in the figure, both two curves are shown with two reflection zeros and in good accordance. More importantly, the simulated impedance bandwidth is $1.6-2.84 \mathrm{GHz}$ with much lower reflection coefficient of $\left|S_{11}\right|<-15 \mathrm{~dB}$, which is also wider than the impedance bandwidth of $\left|S_{11}\right|<-10$ dB shown in Fig. 1 (b).

\section{ANTENNA DESIGN}

With the specially designed TS-TL based power divider, a wideband differentially fed antenna is developed for dual-polarized radiation in this Section. The presented antenna features high in-band selectivity with four reflection zeros, quasi-elliptic filtering property with two controllable radiation nulls and a wideband and high $2^{\text {nd }}$ order harmonic suppression.

\section{A. Configuration}

Fig. 4 shows the configuration of the presented wideband differentially fed dual-polarized antenna by using the TS-TL based power divider. As shown in Fig. 4 (a), the presented antenna is composed of a radiator printed on a substrate, four coaxial cables for the direct differential excitation, and a square copper sheet with the length $\mathrm{Lg}$ and distance $\mathrm{H}$ as the planar reflector. The commercially available substrate of Rogers $4003 \mathrm{C}$ with the thickness of $0.813 \mathrm{~mm}$ and relative permittivity of 3.55 is used in the antenna radiator design. Note that in the antenna design, the input port pair of port 1 and port 3 is defined as differential port $\mathrm{d} 1$ for $-45^{\circ}$ polarization, and the input port pair of port 2 and port 4 is defined as differential port $\mathrm{d} 2$ for $+45^{\circ}$ polarization. 


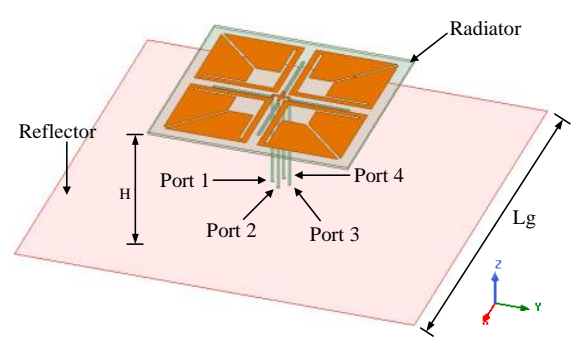

(a)

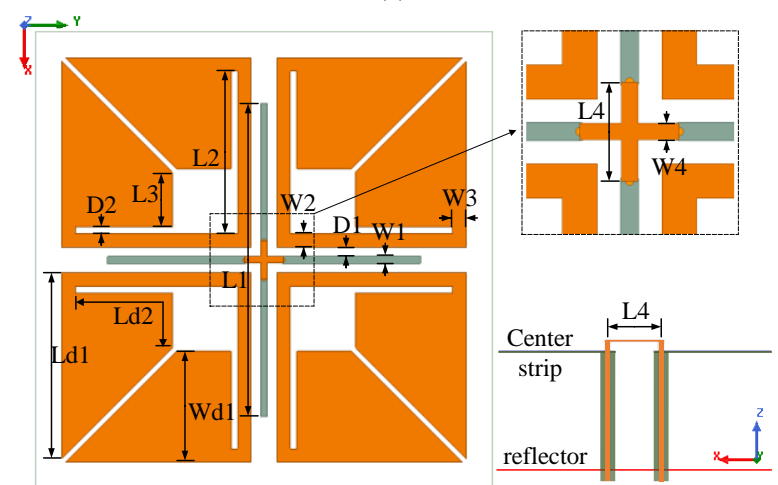

(b)

Fig. 4. Configuration of the presented differentially fed dual-polarized antenna by using the TS-TL based power divider. (Detailed parameters in the configuration. $\mathrm{Lg}=140 \mathrm{~mm}, \mathrm{H}=33 \mathrm{~mm}, \mathrm{~W} 1=1.1 \mathrm{~mm}, \mathrm{D} 1=1.4 \mathrm{~mm}, \mathrm{~W} 2=2.1$ $\mathrm{mm}, \mathrm{L} 1=48 \mathrm{~mm}, \mathrm{~L} 2=25 \mathrm{~mm}, \mathrm{~W} 3=2.1 \mathrm{~mm}, \mathrm{~L} 3=8.4 \mathrm{~mm}, \mathrm{D} 2=1 \mathrm{~mm}, \mathrm{Wd} 1=17$ $\mathrm{mm}, \mathrm{Ld} 1=28.5 \mathrm{~mm}, \mathrm{Ld} 2=23.3 \mathrm{~mm}, \mathrm{~W} 4=1 \mathrm{~mm}, \mathrm{~L} 4=6 \mathrm{~mm}$.)

Fig. 4 (b) shows the detailed configuration of the antenna radiator. It can be seen that only one substrate is used for the antenna design with simple configuration. The top copper layer of the substrate is shown in orange colour, and the bottom copper layer is shown in navy colour. The radiator is composed of the TS-TL based power divider in the center and four dipoles at the four sides with the length Ld1 and width Wd1 for dual-polarization. In the center of the power divider, four coaxial cables are soldered for the differential excitation. The outer conductor of the coaxial cable is soldered to the center strip of the TS-TL, while the inner conductor of the coaxial cable is penetrating through the center strip of the TS-TL without the electrical connection, and then it is soldered to the crossed copper strip on the top layer of the substrate. The detailed soldering method of the coaxial cables and the crossed feed line on the top layer of the substrate are shown in the right bottom inset figure of Fig. 4 (b). All the detailed parameters in the configuration are given in the caption of Fig. 4 for the designer's reference.

\section{B. Multi-Resonance}

The presented differentially fed dual-polarized antenna has wide impedance bandwidth for the simulated $\left|S_{d d 11}\right|<-15 \mathrm{~dB}$ covering 1.65-2.91 GHz with four resonances to ensure high in-band selectivity. As the red solid curve shown in Fig. 5 (a), four reflection zeros are clearly observed at $1.72 \mathrm{GHz}\left(f_{1}\right), 2.14 \mathrm{GHz}\left(f_{2}\right), 2.64$ $\mathrm{GHz}\left(f_{3}\right)$, and $2.88 \mathrm{GHz}\left(f_{4}\right)$. These four resonances are produced by the center TS-TL based power divider and the connected dipoles respectively. As illustrated in Section II. A, the TS-TL based power divider can introduce two reflection zeros when the TS-TL works as the impedance inverter. Therefore, the additional two resonances will be introduced by the connected dipoles.

Fig. 5 shows the reflection coefficient of the presented differentially fed antenna varies with the length (Ld1) and width (Wd1) of the dipole. As shown in Fig. 5 (a), when the dipole length is increased from $28 \mathrm{~mm}$ to $29 \mathrm{~mm}$, only the second resonance $\left(f_{2}\right)$ shifts obviously to the lower frequency, which causes the reflection coefficient in the center being deteriorated. This denotes that the second resonance is introduced by the length of the dipole. The effect of the dipole width is studied in Fig. 5 (b). As can be seen, only the fourth resonance $\left(f_{4}\right)$ changes obviously as the variance of Wd1. The working frequency band is narrowed as the increase of $\mathrm{Wd1}$. Therefore, the fourth resonance is mainly controlled by the width of the dipole.

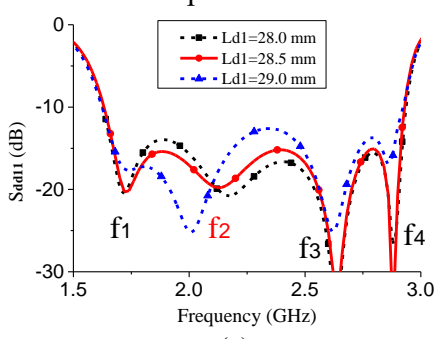

(a)

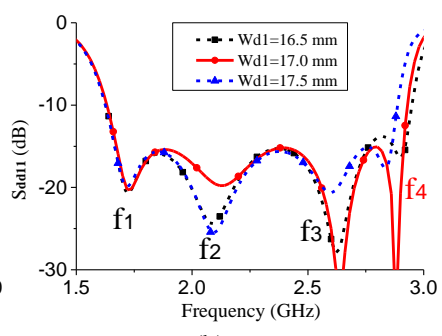

(b)
Fig. 5. Reflection coefficient and input impedance of the presented antenna vary with different parameters. (a) Length of the dipole (Ld1). (b) Width of the dipole (Wd1).

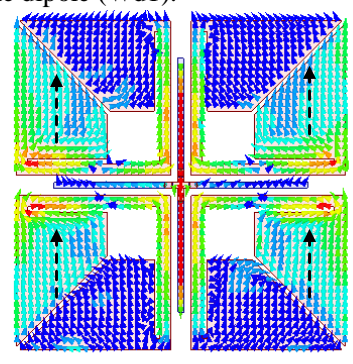

(a)

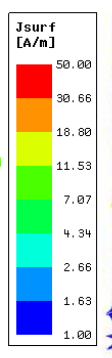

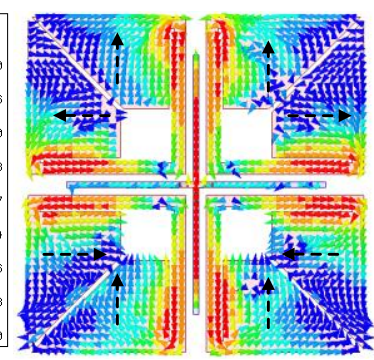

(b)
Fig. 6. Current distributions on the surface of the dipoles at (a) $2.14 \mathrm{GHz}\left(f_{2}\right)$ and (b) $2.88 \mathrm{GHz}\left(f_{4}\right)$.

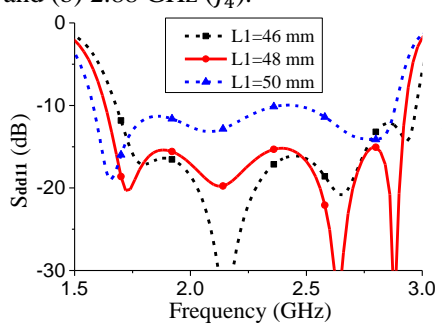

(a)

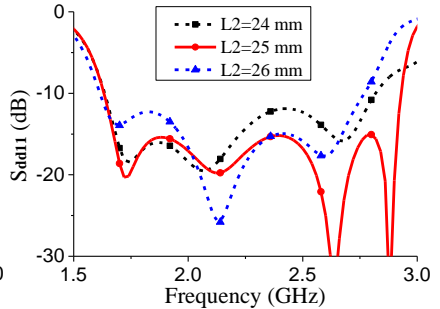

(b)
Fig. 7. Reflection coefficient of the presented antenna varies with different parameters. (a) Lengths of the center strip (L1) and (b) the outer strip (L2) of the TS-TL.

It should be noted that two resonances are in relation to the dipole, but they resonate on the different parts of the dipoles. This can be illustrated by the current distribution on the surface of four dipoles. As shown in Fig. 6, when the surface current is excited at $f_{2}$, strong current magnitude is observed on the left and right dipoles along the length direction, and the collective direction is vertical. Whereas when the surface current is excited at $f_{4}$, strong current magnitude is observed on the four dipole arms along the width direction. The collective current direction on the four dipoles is also vertical. Therefore, these two resonances work for the same polarization.

The parameters of the TS-TL based power divider can have the significantly effects on the return loss of the antenna. As shown in Fig. 7 (a), the frequencies of the first and fourth reflection zeroes are sensitive to the length of the center strip, which vary a lot as compared to the second and the third reflection zeroes. In addition, the reflection at the input port becomes higher and the whole 
working frequency band will shift to the lower frequency as the increase of L1. In Fig. 7 (b), the frequencies of the third and fourth reflection zeroes change a lot as the increase of L2. The bandwidth becomes narrowed no matter when L2 is increased or decreased, which denotes that $\mathrm{L} 2=25 \mathrm{~mm}$ is the optimal value for the presented antenna. Therefore, as a key component for the presented antenna, when the parameters on the TS-TL based power divider are varied, more reflection zeros will be affected, and the related reflection coefficients will also be affected.

\section{Controllable Radiation Nulls}

The presented antenna has two controllable radiation nulls at the both lower and upper out-of-bands with quasi-ellipse filtering response. Along with the wideband $2^{\text {nd }}$-order harmonic suppression owing to the differentially fed method, the presented antenna can have high suppression over the undesired lower out-of-band (0.6-1 $\mathrm{GHz}$ ) and upper out-of-band (3.3-5 GHz). Fig. 8 (a) shows the peak realized gain of the antenna varies with different feed positions (L4). It can be seen that, as the increase of the feed distance between the differentially fed coaxial cables, the radiation null at the lower outof-band moves substantially to the upper frequency. It also can be noted that the gain bandwidth is narrowed and the radiation null at the upper out-of-band is deepened as the increase of L4. This denotes that the feed position can significantly affect the input impedance of the antenna.

To illustrate the inner mechanism of the first radiation null, the surface current distribution on inner strip of the TS-TL and the outer conductor of the coaxial cables at the frequency of the first radiation null $(0.9 \mathrm{GHz})$ is shown in Fig. 8 (b). It can be seen that the current is symmetrically distributed on the center strips of TS-TL and outer conductors of the coaxial cables, and the current is quarter wavelength distributed along their surfaces. Therefore, the related equivalent circuit at the driven point $\mathrm{A}$ can be obtained and shown in Fig. 8 (c). In the equivalent circuit, $L_{S c}$ is the equivalent inductor caused by the shorted coaxial cables, $C_{O S}$ is the equivalent capacitor caused by the open-circuited center strip. Therefore, the input admittance at the driven port A can be calculated as

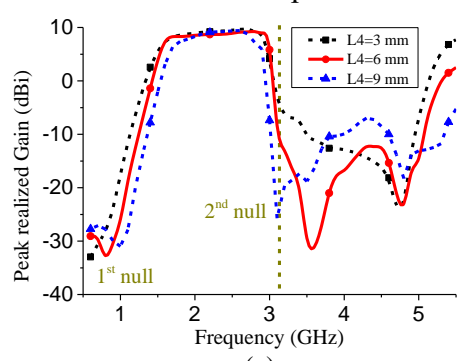

(a)

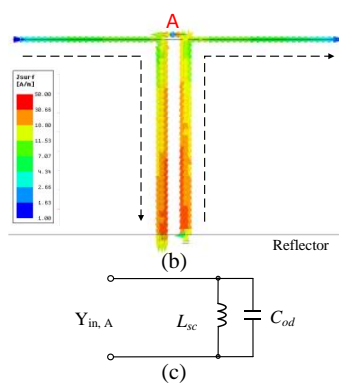

(c)
Fig. 8 (a). Peak realized gain of the presented antenna varies with different feed positions (L4). (b) Surface current distribution on the center strip of the TS-TL and outer conductor of the coaxial cables at the frequency of the first radiation null. (c) Equivalent circuit of the structure at the driven point A.

$$
\begin{aligned}
Y_{i n, A} & =j \omega C_{o s}+1 /\left(j \omega L_{s c}\right) \\
& =\frac{1-\omega^{2} L_{s c} C_{o s}}{j \omega L_{s c}}
\end{aligned}
$$

When the structure shown in Fig. 8 (b) is resonated, $Y_{i n, A}=0$, the input impedance at the driven point $\mathrm{A}$ will become infinite, and coaxial cables will be unloaded. As this frequency is caused by the resonance of the overall length of the center strip of TS-TL and outer conductor of coaxial cable. Because of the quarter wavelength current distribution, the corresponding resonant frequency can be estimated as

$$
f_{\text {null }, 1} \approx \frac{c}{4 H+2(L 1-L 4)}
$$

According to (3), the first radiation null at the lower out-of-band can be designed by elaborately changing the feed position of the coaxial cables.

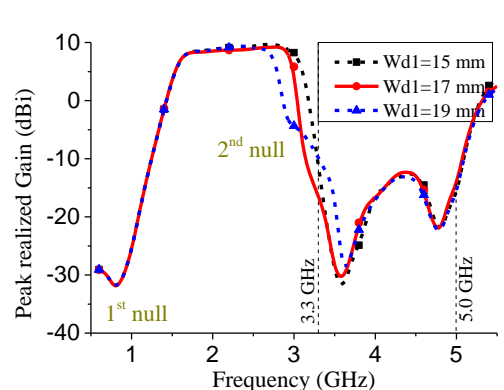

(a)

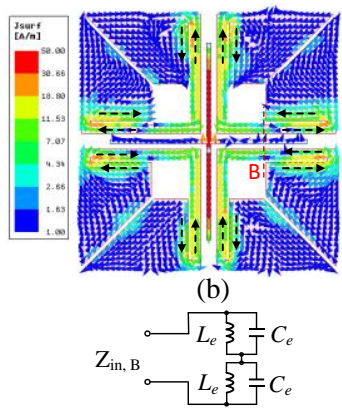

(c)
Fig. 9. (a) Peak realized gain of the presented antenna varies with different widths of the dipole (Wd1). (b) Surface current distribution on the proposed antenna at the frequency of the second radiation null. (c) Equivalent circuit of the structure at the reference point $B$.

Fig. 9 (a) shows the peak realized gain of the antenna varies with different widths of the dipole (Wd1). As the increase of $\mathrm{Wd} 1$, the upper radiation null moves from the upper frequency to the lower frequency. It should be noted that, because of the effect of the wideband and high $2^{\text {nd }}$-order harmonic suppression, the upper radiation null will be overshadowed by the $2^{\text {nd }}$-order harmonic suppression when the dipole width is too small. As shown in the figure, the second radiation null is submerged into the harmonic suppression when $\mathrm{Wd} 1=15 \mathrm{~mm}$. However, when the dipole width is increased to $19 \mathrm{~mm}$, the radiation null will be clearly observed. To get the high suppression in the frequency band of $3.3-5 \mathrm{GHz}$, especially high suppression in the two $5 \mathrm{G}$ sub- $6 \mathrm{GHz}$ frequency bands $(3.3-3.6 \mathrm{GHz}$ and $4.8-5 \mathrm{GHz}), \mathrm{Wd} 1=17 \mathrm{~mm}$ is chosen in the antenna design with the radiation null at around $3.2 \mathrm{GHz}$.

The surface current distribution on the proposed antenna at the radiation null of $3.2 \mathrm{GHz}$ is shown in Fig. 9 (b) to illustrate the inner mechanism of this radiation null. As can be seen in the figure, strong current magnitude only exists around the slots formed by the dipole arm and the outer strip of TS-TL, and reverse current pairs are observed on these inner edges. This denotes that the second radiation null at the upper out-of-band is introduced by these resonant slots. Fig. 9 (c) shows the equivalent circuit at the reference point $B$ on one of the quarter parts of the radiator. In the equivalent circuit, $L_{e}$ is the equivalent inductor caused by the current flowing along the edges of the dipole arm and TS-TL, and $C_{e}$ is the equivalent capacitor caused by the strong coupling between the dipole arm and the outer strip of TS-TL. Then, the input impedance at the reference point $B$ can be calculated as

$$
Z_{i n, B}=\frac{j 2 \omega L_{e}}{1-\omega^{2} L_{e} C_{e}}
$$

When the circuit is resonating, $Z_{i n, B}$ will become infinite, this means the input impedance at the reference point $\mathrm{B}$ will become open-circuited. Therefore, the TS-TL based power divider will be unloaded, and the dipoles will be unexcited. Because this resonance is caused by the slot resonance formed by the dipole arm and outer strip of TS-TL, the related null frequency can be estimated as

$$
f_{\text {null }, 1} \approx \frac{c}{4(W d 1-W 3)}
$$

Based on (5), the second radiation null at the upper out-of-band can be designed by elaborately designing the width of the dipole arm. 


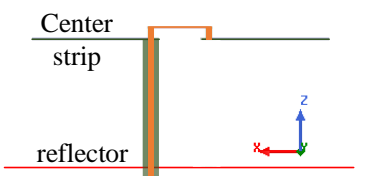

(a)

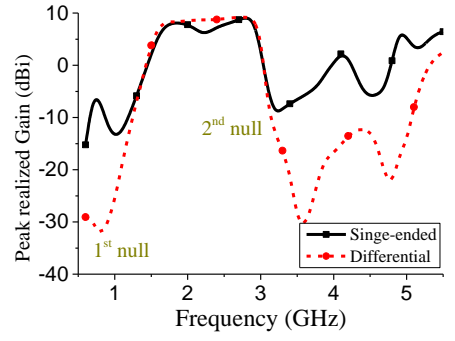

(b)
Fig. 10. Peak realized gain of the presented antenna compared to the singleended counterpart. (a) Side view of the single-ended counterpart. (b) Comparison results.

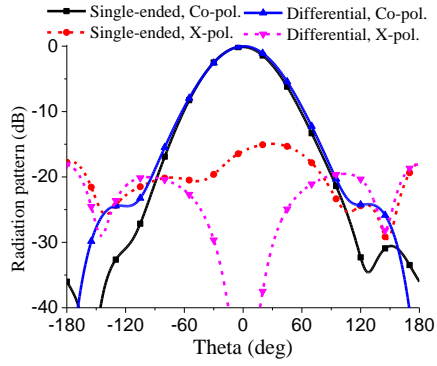

(a)

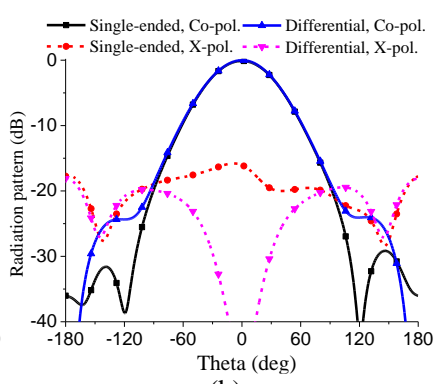

(b)
Fig. 11. Normalized radiation patterns of the presented antenna compared to the single-ended counterpart at $2.2 \mathrm{GHz}$ when $+45^{\circ}$ polarization is excited. (a) xz-plane (b) yz-plane.

By using the differentially fed method, an obvious advantage is that a wideband and high $2^{\text {nd }}$-order harmonic suppression can be obtained at the upper out-of-band. A single-ended antenna counterpart is simulated and shown in Fig. 10 (a) to illustrate this effect. In the figure, only one coaxial cable is used to excite the radiator with the outer conductor of the coaxial cable connected to one center strip of TS-TL and the inner conductor connected to the opposite center strip of TS-TL. As shown in Fig. 10 (b), two radiation nulls beside the gain bandwidth can be clearly observed even without the differentially fed cable pairs, this proves the effectiveness of the introduction of these two radiation nulls. By utilizing the differentially fed method, undesired harmonic radiations with the resulted gains of around $5 \mathrm{dBi}$ at about $4.1 \mathrm{GHz}$ and 5.0 $\mathrm{GHz}$ are successfully suppressed. Combining with the introduced second radiation null, steep roll-of-rate at the upper band-edge is achieved, and the resulted simulated peak realized gain from 3.2 $\mathrm{GHz}$ to $5.05 \mathrm{GHz}$ is lower than $-12.3 \mathrm{dBi}$.

Owing to the differentially fed method, another advantage is that a very low cross-polarization level can be achieved. Fig. 11 shows the simulated normalized radiation patterns of the presented antenna compared to the single-ended counterpart at $2.2 \mathrm{GHz}$ when $+45^{\circ}$ polarization is excited. As can be seen the figure, the presented antenna fed by different methods can obtain almost the same radiation characteristic for the co-polarized radiation patterns, especially in the main-beam direction. However, difference can be observed between the cross-polarized radiation patterns. The crosspolarization level for the presented differentially fed antenna in the main-beam direction is very ideal and symmetrical, and its value at theta $=0^{\circ}$ is close to $-\infty$. Whereas high and asymmetrical crosspolarization level is observed for the single-ended counterpart in the main-beam direction, and the simulated cross-polarized radiation patterns in both two planes at theta $=0^{\circ}$ are only $16 \mathrm{~dB}$ lower than the co-polarized radiation patterns.

\section{Design Guidelines}

Based on the above analysis and illustration, to guide the design of the presented wideband differentially fed dual-polarized antenna, key design guidelines are concluded as below.

1. Design a wideband TS-TL based power divider by using analysis in Section II before the design of the presented antenna.

2. Select the proper feed position of the differential coaxial cable pair to design the first radiation null at the required lower out-ofband according to (3).

3. Design the proper length of the slot formed by the dipole arm and the outer strip of TS-TL to realize the second radiation null at the required upper out-of-band using (5).

4. Finely adjust the parameters of the TS-TL based power divider and the connected dipole to realize wide impedance bandwidth with four reflection zeros within the band of interest, and a high $2^{\text {nd }}$-order harmonic suppression will be achieved.

5. Finely adjust the feed position the coaxial cable pair and the slot length to achieve the required radiation nulls with the quasi-elliptic response.

6. Steps 4 and 5 may be needed several rounds of fine adjustments to achieve the final expected antenna performances.

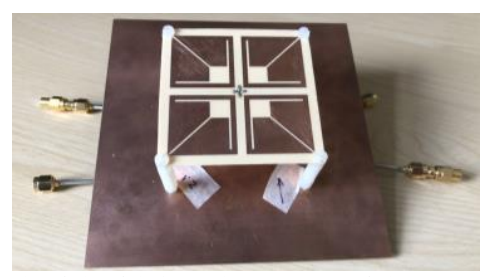

Fig. 12. Photograph of the fabricated prototype of the presented differentially fed dual-polarized antenna.

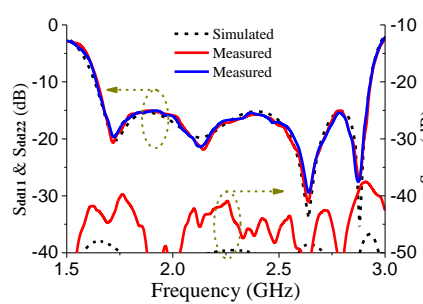

(a)

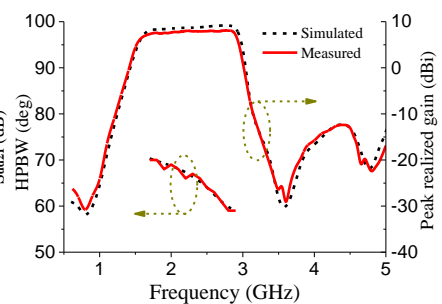

(b)
Fig. 13 (a). Measured and simulated differential S-parameters of the presented differentially fed dual-polarized antenna. (b) Measured and simulated peak realized gains and H-plane HPBWs of the fabricated prototype when $+45^{\circ}$ polarization is excited.

\section{RESULTS AND DisCUSSION}

The presented antenna was designed and fabricated for the verification of all the antenna performance. Fig. 12 shows the photograph of the fabricated prototype of the presented antenna. The measured differentially fed S-parameters are shown in Fig. 13 (a), which is measured by using Anritsu 37397C vector network analyzer. As shown in the figure, the measured S-parameters agree well with the simulated results. Four reflection zeroes are measured with high in-band selectivity, and the overlapped impedance bandwidth for both $\left|S_{d d 11}\right|$ and $\left|S_{d d 22}\right|$ lower than $-15 \mathrm{~dB}$ is from $1.67 \mathrm{GHz}$ to 2.91 $\mathrm{GHz}$. The measured impedance bandwidth is slightly narrowed as compared to the simulated results. Owing the symmetrical differentially fed method, the measured isolation between the two differential port pair is higher than $37.5 \mathrm{~dB}$.

Fig. 13 (b) shows the measured peak realized gain and H-plane half-power beamwidth (HPBW) when $+45^{\circ}$ polarization is excited, which are also compared with the simulated results. The measured differentially fed in-band peak realized gain is 7.3-8.3 dBi, and the 
out-of-band gain from $3.3 \mathrm{GHz}$ to $5 \mathrm{GHz}$ is lower than $-12.3 \mathrm{dBi}$. Suppressions in the two $5 \mathrm{G}$ bands of $3.3-3.6 \mathrm{GHz}$ and $4.8-5 \mathrm{GHz}$ are high than $24.8 \mathrm{~dB}$ and $25.2 \mathrm{~dB}$ respectively. The measured H-plane $\mathrm{HPBW}$ is $59-70^{\circ}$ within the bandwidth.

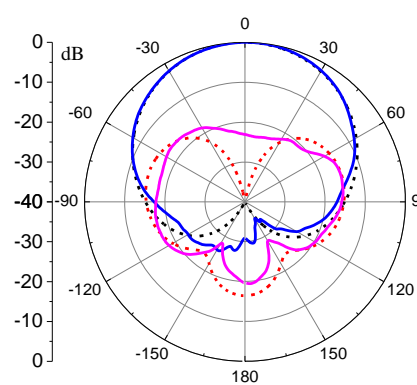

(a)

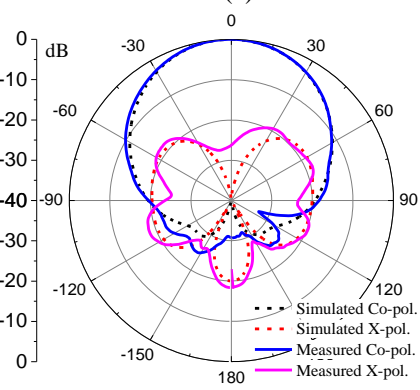

(c)

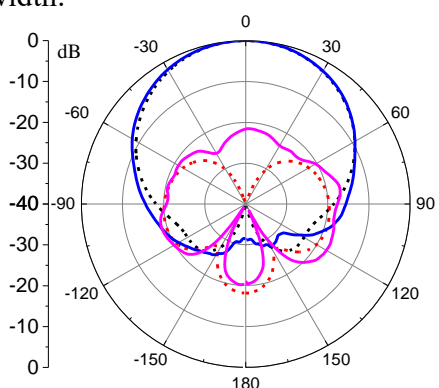

(b)
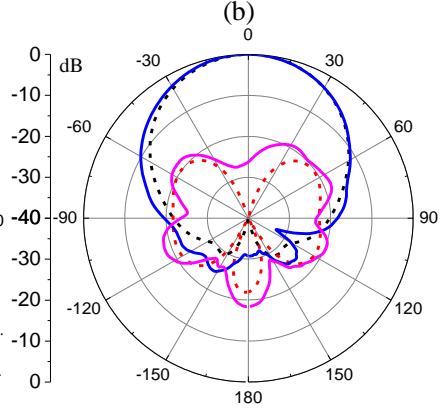

(d)
Fig. 14. Measured and simulated normalized radiation patterns in H-plane when $-45^{\circ}$ polarization is excited at the frequencies of (a) $1.7 \mathrm{GHz}$, (b) 2.2 $\mathrm{GH}$, (c) $2.7 \mathrm{GHz}$, and (d) $2.9 \mathrm{GHz}$.

The measured and simulated normalized H-plane radiation patterns at four different frequencies are shown in Fig. 14. Owing to the symmetry of the antenna, only radiation patterns excited for $-45^{\circ}$ polarization in H-plane are provided in the figure for brevity. As can be seen, good accordance is achieved between the measured and simulated radiation patterns. The measured cross-polarization level is very low, which is $21.6 \mathrm{~dB}$ lower than the co-polarization level in the broadside direction, and $16.1 \mathrm{~dB}$ lower than the co-polarization level in the $\pm 30^{\circ}$ beamwidth range. The measured front to back ratio is higher than $16.4 \mathrm{~dB}$ within the bandwidth. The slight discrepancies between the simulated and measured radiation patterns may be caused by the position errors of the antenna and the blockage of the feed coaxial cables.

\section{CONCLUSION}

This communication presents a novel method to design a wideband differentially fed dual-polarized antenna by using TS-TLs. A power divider composed of four TS-TLs is firstly presented and illustrated by using the equivalent circuit analysis. It shows that the proposed power divider can have a wide bandwidth under differential excitation. Then a wideband differentially fed dualpolarized is developed by using the proposed power divider. It shows that the presented antenna can have a wide impedance bandwidth with four resonances, and wideband and high $2^{\text {nd }}$-order suppression over two $5 \mathrm{G}$ sub- $6 \mathrm{GHz}$ frequency bands. Finally, the presented differentially fed dual-polarized antenna was designed, fabricated, and measured for verification. Both the simulated and measured results demonstrate that the presented antenna can be a very competitive candidate for the current base station applications.
[1] S. Gao, L. W. Li, M. S. Leong, and T. S. Yeo, "A broad-band dualpolarized microstrip patch antenna with aperture coupling," in IEEE Trans. Antennas Propag., vol. 51, no. 4, pp. 898-900, April 2003.

[2] H. Lai and K. Luk, "Dual polarized patch antenna fed by meandering probes," IEEE Trans. Antennas Propag., vol. 55, no. 9, pp. 2625-2627, Sept. 2007.

[3] Y. Guo, K. Khoo, and L. C. Ong, "Wideband dual-polarized patch antenna with broadband baluns," IEEE Trans. Antennas Propag., vol. 55, no. 1, pp. 78-83, Jan. 2007.

[4] Y. Wang and Z. Du, "Dual-polarized slot-coupled microstrip antenna array with stable active element pattern," IEEE Trans. Antennas Propag., vol. 63, no. 9, pp. 4239-4244, Sept. 2015.

[5] C. Mao, S. Gao, Y. Wang, F. Qin, and Q. Chu, "Multimode resonatorfed dual-polarized antenna array with enhanced bandwidth and selectivity," IEEE Trans. Antennas Propag., vol. 63, no. 12, pp. 54925499, Dec. 2015.

[6] K. Wong and T. Chiou, "Broadband dual-polarized patch antennas fed by capacitively coupled feed and slot-coupled feed," IEEE Trans. Antennas Propag., vol. 50, no. 3, pp. 346-351, March 2002.

[7] X. Y. Zhang, W. Duan, and Y. Pan, "High-gain filtering patch antenna without extra circuit," IEEE Trans. Antennas and Propag., vol. 63, no. 12, pp. 5883-5888, Dec. 2015.

[8] Y. Cui, R. Li, and H. Fu, "A broadband dual-polarized planar antenna for 2G/3G/LTE base stations," IEEE Trans. Antennas Propag., vol. 62, no. 9, pp. 4836-4840, Sept. 2014.

[9] Y. Luo, Q. Chu, and D. Wen, "A plus/minus 45 degree dual-polarized base-station antenna with enhanced cross-polarization discrimination via addition of four parasitic elements placed in a square contour," IEEE Trans. Antennas Propag., vol. 64, no. 4, pp. 1514-1519, April 2016.

[10] H. Huang, Y. Liu, and S. Gong, "A novel dual-broadband and dualpolarized antenna for $2 \mathrm{G} / 3 \mathrm{G} / \mathrm{LTE}$ base stations," IEEE Trans. Antennas Propag., vol. 64, no. 9, pp. 4113-4118, Sept. 2016.

[11] L. Ge and K. Luk, "Linearly polarized and dual-polarized magnetoelectric dipole antennas with reconfigurable beamwidth in the H-plane," IEEE Trans. Antennas Propag., vol. 64, no. 2, pp. 423-431, Feb. 2016.

[12] S. Zhou, Z. Peng, G. Huang, and C. Sim, "Design of a novel wideband and dual-polarized magnetoelectric dipole antenna," IEEE Trans. Antennas Propag., vol. 65, no. 5, pp. 2645-2649, May 2017.

[13] L. Wen et al., "A compact wideband dual-polarized antenna with enhanced upper out-of-band suppression," IEEE Trans. Antennas Propag., vol. 67, no. 8, pp. 5194-5202, Aug. 2019.

[14] C. Ding, H. Sun, R. W. Ziolkowski, and Y. Jay Guo, "A dual layered loop array antenna for base stations with enhanced cross-polarization discrimination," IEEE Trans. Antennas Propag., vol. 66, no. 12, pp. 6975-6985, Dec. 2018.

[15] C. F. Ding, X. Y. Zhang, Y. Zhang, Y. M. Pan, and Q. Xue, "Compact broadband dual-polarized filtering dipole antenna with high selectivity for base-station applications," IEEE Trans. Antennas Propag., vol. 66, no. 11, pp. 5747-5756, Nov. 2018.

[16] Y. Liu, S. Wang, N. Li, J. Wang, and J. Zhao, "A compact dual-band dual-polarized antenna with filtering structures for sub-6 ghz base station applications," IEEE Antennas Wireless Propag. Lett., vol. 17, no. 10, pp. 1764-1768, Oct. 2018.

[17] H. Hu, F. Chen, J. Qian, and Q. Chu, "A differential filtering microstrip antenna array with intrinsic common-mode rejection," IEEE Trans. Antennas Propag., vol. 65, no. 12, pp. 7361-7365, Dec. 2017.

[18] K. Sun, Y. Zhao, D. Yang and J. Pan, "A single-layer differential substrate-integrated slot antenna with common-mode rejection," IEEE Antennas Wireless Propag. Lett., vol. 18, no. 2, pp. 392-396, Feb. 2019.

[19] L. Wen et al., "A wideband differentially fed dual-polarized antenna with wideband harmonic suppression," IEEE Trans. Antennas Propag., vol. 67, no. 9, pp. 6176-6181, Sept. 2019.

[20] Y. Cui, X. Gao, and R. Li, "A broadband differentially fed dualpolarized planar antenna," IEEE Trans. Antennas Propag., vol. 65, no. 6, pp. 3231-3234, June 2017.

[21] Z. Tang, J. Liu, R. Lian, Y. Li, and Y. Yin, "Wideband differentially fed dual-polarized planar antenna and its array with high common-mode suppression," IEEE Trans. Antennas Propag., vol. 67, no. 1, pp. 131139, Jan. 2019.

[22] L. Wen et al., "A wideband differentially driven dual-polarized antenna by using integrated six-port power divider," IEEE Trans. Antennas Propag., vol. 67, no. 12, pp. 7252-7260, Dec. 2019. 